\title{
Structure of HIV-1 gp41 with its membrane anchors targeted by neutralizing antibodies
}

3 6

Christophe Caillat ${ }^{1} \uparrow$, Delphine Guilligay ${ }^{1} \uparrow$, Johana Torralba ${ }^{2}$, Nikolas Friedrich ${ }^{3}$, Jose L. Nieva ${ }^{2}$, Alexandra Trkola ${ }^{3}$, Christophe Chipot ${ }^{4,5,6}$, François Dehez ${ }^{4,5}$ and Winfried Weissenhorn ${ }^{1 *}$

${ }^{1}$ Univ. Grenoble Alpes, CEA, CNRS, Institut de Biologie Structurale (IBS), 71, avenue des Martyrs, 38000 Grenoble, France.

${ }^{2}$ Biofisika Institute (CSIC, UPV/EHU) and Department of Biochemistry and Molecular Biology, University of the Basque Country (UPV/EHU), 48080, Bilbao, Spain.

${ }^{3}$ Institute of Medical Virology, University of Zurich, 8057 Zurich, Switzerland.

${ }^{4}$ Laboratoire de Physique et Chimie Théoriques (LPCT), University of Lorraine, CNRS, Boulevard des Aiguillettes, 54506 Vandoeuvre-lès-Nancy Cedex, France.

${ }^{5}$ Laboratoire International Associé, CNRS and University of Illinois at Urbana-Champaign, 54506 Vandoeuvre-lès-Nancy Cedex, France.

${ }^{6}$ Department of Physics, University of Illinois at Urbana-Champaign, 1110 West Green Street, Urbana, Illinois 61801, USA.

$\dagger$ These authors contributed equally

*Correspondence to: winfried.weissenhorn@ibs.fr 


\section{Abstract}

The HIV-1 gp120/gp41 trimer undergoes a series of conformational changes in order to catalyze gp41-induced fusion of viral and cellular membranes. Here, we present the crystal structure of gp41 locked in a fusion intermediate state by an MPER-specific neutralizing antibody. The structure illustrates the conformational plasticity of the six membrane anchors arranged asymmetrically with the fusion peptides and the transmembrane regions pointing into different directions. Hinge regions located adjacent to the fusion peptide and the transmembrane region facilitate the conformational flexibility that allows high affinity binding of broadly neutralizing anti-MPER antibodies. Molecular dynamics simulation of the MPER Ab-induced gp41 conformation reveals the transition into the final post-fusion conformation with the central fusion peptides forming a hydrophobic core with flanking transmembrane regions. This, thus, suggests that MPER-specific broadly neutralizing antibodies can block final steps of refolding of the fusion peptide and the transmembrane region, which is required for completing membrane fusion.

\section{Introduction}

Viral fusion proteins catalyze virus entry by fusing the viral membrane with cellular membranes of the host cell, thereby establishing infection. The HIV-1 envelope glycoprotein (Env) is a prototypic class I fusion protein that shares common pathways in membrane fusion with class II and III viral membrane fusion proteins ${ }^{1-4}$. HIV-1 Env is expressed as a gp160 precursor glycoprotein that is cleaved into the fusion protein subunit gp41 and the receptor binding subunit gp120 by host furin-like proteases. Gp41 anchors Env to the membrane and associates noncovalently with gp120, thereby forming a stable trimer of heterodimers, the metastable Env prefusion conformation ${ }^{5,6}$. Orchestration of a series of conformational changes transforms energyrich prefusion Env into the low-energy, highly stable gp41 post-fusion conformation, which provides the free energy to overcome the kinetic barriers associated with bringing two opposing membranes into close enough contact to facilitate membrane fusion ${ }^{2,3}$.

HIV-1 gp41 is composed of several functional segments that have been shown or suggested to extensively refold upon fusion activation: the N-terminal fusion peptide (FP), a fusion peptide proximal region (FPPR), the heptad repeat region 1 (HR1), a loop region followed by HR2, the membrane proximal external region (MPER), the transmembrane region (TMR), and a cytoplasmic domain. Structures of native Env trimers in complex with different broadly neutralizing antibodies revealed the conformation of the gp41 ectodomain lacking MPER in the native prefusion conformation ${ }^{7-12}$. Env interaction with CD4 results in opening of the closed prefusion trimer ${ }^{13,14}$, 
72 which includes the displacement of gp120 variable regions 1 and 2 (V1-V2) at the apex of the trimer

but no changes in gp41 ${ }^{15}$. This is required for the formation of a stable ternary complex of EnvCD4 with the co-receptor ${ }^{16-18}$. Co-receptor binding positions prefusion gp41 closer to the host-cell membrane ${ }^{5}$ and induces a cascade of conformational changes in gp41. First, the fusion peptide is repositioned by $\sim 70 \AA^{9}$ to interact with the target cell membrane, generating a $110 \AA$ extended fusion-intermediate conformation ${ }^{19,20}$ that bridges the viral and the host cell membrane ${ }^{21}$. Subsequent refolding of HR2 onto HR1 leads to the formation of the six-helix bundle core structure 22-24, which pulls the viral membrane into close apposition to the host-cell membrane and, thus, sets the stage for membrane fusion ${ }^{22}$.

Membrane fusion generates a lipid intermediate hemifusion state, that is predicted to break and evolve to fusion pore opening ${ }^{25}$, which is regulated by six-helical bundle formation ${ }^{26,27}$. Furthermore residues within FPPR, FP, MPER and TM have been as well implicated in fusion ${ }^{28-32}$ indicating that final steps in fusion are controlled by the conformational transitions of the membrane anchors into the final post-fusion conformation.

Here, we set out to understand the conformational transitions of the gp41 membrane anchors. We show that the presence of the membrane anchors increases thermostability. However, complex formation with a MPER-specific neutralizing nanobody induced an asymmetric conformation of the membrane anchors, which constitutes a late fusion intermediate. We show that this conformation can be targeted by MPER bnAbs consistent with the possibility that MPERspecific nAbs can interfere all along the fusion process until a late stage. Starting from the asymmetric conformation, we used MD simulation based modelling to generate the final postfusion conformation, which reveals a tight helical interaction of FP and TM in the membrane consistent with its high thermostability. Our work, thus, elucidates the structural transitions of the membrane anchors that are essential for membrane fusion, which can be blocked by MPER-specific bnAbs up to a late stage in fusion.

\section{Results}

\section{Gp41FP-TM interaction with 2 H10.}

Two gp41 constructs, one containing residues 512 to 581 comprising FP, FPPR and HR1 (Nterminal chain, chain $\mathrm{N}$ ) and one coding for resides 629 to 715 including HR2, MPER and TM (Cterminal chain, chain C)( Fig. S1A) were expressed separately, purified and assembled into the monodisperse trimeric complex gp41FP-TM (Fig. S1B). Gp41FP-TM reveals a thermostability of $>95^{\circ} \mathrm{C}$ as measured by circular dichroism (Fig. S2A) indicating that the presence of FP and TMR 
increases the thermostability by $>7^{\circ} \mathrm{C}$ compared to gp 41 lacking FP and $\mathrm{TM}^{33}$. In order to facilitate crystallization, gp41FP-TM was complexed with the llama nanobody $2 \mathrm{H} 10^{34}$ in $\beta$-OG buffer and purified by size exclusion chromatography (SEC)(Fig. S1C). To determine the stoichiometry of binding, we performed isothermal titration calorimetry (ITC), which indicated that gp41FP-TM and 2H10 form a 3:1 complex with a $\mathrm{K}_{\mathrm{D}}$ of $2.1+/-0.9 \mu \mathrm{M}$ (Fig. S2B). Interaction of gp41FP-TM with 2H10 was further confirmed by biolayer interferometry (BLI) analysis (Fig. S2C).

\section{Crystal structure of gp41 in complex with $2 \mathrm{H10}$}

The structure of gp41FP-TM in complex with $2 \mathrm{H} 10$ was solved by molecular replacement to a resolution of $3.8 \AA$ (Table S1). The asymmetric unit contained trimeric gp41FP-TM bound to one 2H10 nanobody as indicated by ITC (Fig. S2B). The six-helix bundle structure composed of three $\mathrm{N}$-terminal and three C-terminal chains is conserved from HR1 residue A541 to HR2 residue L661 in all three protomers, and identical to previous structures ${ }^{22,23}$. However, TMR and FP do not follow the three-fold symmetry and their chains point into opposite directions (Fig. 1A). 2H10 interacts with chain C-A (Fig. 1A and B) and induces a partially extended MPER conformation, including a kink at L669 that positions the rest of MPER and TM (N674 to V693) at a $45^{\circ}$ angle with respect to the six-helix threefold symmetry axis. The corresponding N-terminal chain A (chain N-A) has its FP disordered and FPPR from G527 to A533 is flexible, while the remaining FPPR and HR1 form a continuous helix (Fig. 1C). The chain C-A 2H10 epitope spans from residues Q658 to N671, which is involved in a series of polar contacts with $2 \mathrm{H} 10$. These include interactions of gp41FPTM E662 to 2H10 Y37, S668 and the carbonyl of D664 to R56, K665 to E95, N671 and the carbonyl of A667 to R54, K655 to R97 and R93 contacts E95 to position it for interaction with K665 (Fig. 1B). Notably, mutations of R56, R93, E95 and R97 have been shown to affect interaction ${ }^{34}$. Chain N-B of the second protomer forms a long continuous helix comprising FP, FPPR and HR1 from residues L518 to D589 with the first six residues of FP being disordered. Likewise, chain C-B folds into a continuous helix from M629 to A700 comprising HR2, MPER and TM (Fig. 1D). C $\alpha$ superimposition of chain C-B with MPER containing gp41 structures ${ }^{33,35}$ yields root mean-square deviations of $0.55 \AA$ and $0.29 \AA$ (Fig. S3), indicating that the straight helical conformation is the preferred conformation in threefold symmetrical gp 41 . In the third protomer, chain N-C has a helical FP linked by flexible residues G531 to A533 to a short helix of FPPR that bends at A541 with respect to helical HR1. Its corresponding chain C-C contains helical HR2 and a flexible region from N671 to N674, which stabilizes a $\sim 45^{\circ}$ rotation of the remaining MPER-TM helix that extends to residue R707 (Fig. 1E). Thus, the structure reveals flexible regions within FPPR and MPER. FPPR flexibility is supported by strictly conserved G528 and G531, while 
MPER has no conserved glycine residues. However, the same kink within L661 to F673 has been observed in the MPER peptide structure ${ }^{36}$, and in complex with bnAb $10 \mathrm{E} 8^{37}$. The N-terminal FP residues 512 to 517 are disordered within the detergent micelle. Flexibility of this region in the absence of membrane is consistent with NMR peptide structures that propose a flexible coil structure of the N-terminal part of FP in solution followed by a helical region starting at L518 ${ }^{38}$ as observed here. Based on the flexible linkage of FP and TMR, we propose that both FPPR and MPER act as hinges during gp41 refolding leading to membrane fusion.

\section{MD simulation of gp41FP-TM in a lipid bilayer}

In order to test whether the structure is influenced by the presence of the detergent, we probed its stability by MD simulation in a bilayer having the lipid composition of the HIV-1 envelope. This confirmed that the structure is stable in a membrane environment during a $1 \mu$ s simulation as only the flexibly linked FP of chain N-C moves within the bilayer during the simulation (Fig. S4A and B). The tip of the 2H10 CDR3 dips into the bilayer (Fig. S4B), hence confirming the membraneanchoring role of $\mathrm{W} 100$ for neutralization ${ }^{34}$.

\section{Neutralization activity of $2 \mathrm{H10}$ depends on membrane interaction}

The structure suggests that $2 \mathrm{H} 10$ induced the asymmetry within the membrane anchors. Crystal packing effects on the conformation can be excluded, because only the $\mathrm{C}$-terminus of the chain $\mathrm{C}$ $\mathrm{C}$ helix is involved in crystal lattice contacts (Fig. S5). We therefore further evaluated $2 \mathrm{H} 10$ as a neutralizing nanobody, which showed modest neutralization as a bi-head (bi-2H10), whereas neutralization depended on W100 located at the tip of CDR3 ${ }^{34}$, a hall mark of MPER-specific bnAbs ${ }^{39}$. In order to improve the breadth and potency of monovalent $2 \mathrm{H} 10$, we increased its membrane interaction capacity by changing CDR3 S100d to F (2H10-F) alone and in combination with additional basic residues S27R, S30K and S74R (2H10-RKRF) within the putative 2H10 membrane-binding interface suggested by MD simulation (Fig. S4C). Wild type 2H10 did not show significant neutralization against a panel of 10 clade B pseudo-viruses as shown previously ${ }^{34}$, with the exception of some weak neutralization of NL4-3 and SF163P3. However, both 2H10-F and 2H10-RKRF show improved potency and breadth neutralizing six and eight pseudo-viruses, respectively, albeit with less potency than wild-type bi-2H10 and bnAb 2F5, the latter recognizing an overlapping epitope (Table 1). This result, thus, confirms monovalent 2H10 as a modest antiMPER Ab that neutralizes by engaging MPER and the membrane. 
The efficacy of bi-2H10 and 2H10-RKRF for blocking membrane merging was further assessed in peptide-induced lipid-mixing assays, whereas a vesicle population is primed for fusion by addition of the N-MPER peptide containing the $2 \mathrm{H} 10$ epitope, which produces a fluorescence intensity spark at time $20 \mathrm{~s}$ (Fig. 2A) ${ }^{40}$. Under these experimental conditions, incorporation of the peptide into the vesicles takes less than $10 \mathrm{sec}^{40}$. After $120 \mathrm{sec}$, the mixture was supplemented with target vesicles fluorescently labeled with N-NBD-PE/N-Rh-PE (indicated by the arrow in Fig. 2A). The increase in NBD intensity as a function of time followed the mixing of the target vesicle lipids with those of the unlabeled vesicles (kinetic trace labeled ' $+\mathrm{N}-\mathrm{MPER}$ '). The increase in NBD fluorescence was not observed when labeled target vesicles were injected in a cuvette containing unlabeled vesicles not primed with peptide ('no peptide' trace). Lipid mixing was strongly attenuated when the vesicles primed for fusion with N-MPER were incubated with bi-2H10 before addition of the target vesicles (Fig. 2A, +N-MPER/+bi-2H10, dotted trace). Thus, the N-MPER-induced membrane perturbations, which can induce fusion with target membranes, were inhibited by incubation with bi-2H10. Comparison of the kinetics of the lipid-mixing blocking effect of 2H10-RKRF, bi-2H10 and the 2F5 Fab showed that the three antibodies inhibited both the initial rates and final extents of lipid mixing induced by N-MPER (Fig. 2B). Using a control MPER peptide lacking the 2H10 and 2F5 epitopes for vesicle priming no inhibition of lipid mixing by 2H10-RKRF, bi-2H10 and 2F5 Fab was observed (Fig. 2C), indicating that the inhibitory effects depend on epitope recognition. Fusion inhibition levels estimated as a function of the antibody concentration further confirmed the apparent higher potency exhibited by the bi-2H10 (Fig. 2D). Lower concentrations of bi-2H10 compared to $2 \mathrm{H} 10-\mathrm{RKRF}$ were required to attain full blocking of the lipid-mixing process when measured $20 \mathrm{sec}$ (initial rates) or $300 \mathrm{sec}$ (final extents) after target-vesicle injection (Fig. 2D and E). The higher inhibitory potency of bi-2H10 indicates an avidity effect, which was also evident when the concentration of the epitope-binding fragments was plotted (Fig. 2D and E, empty squares and dotted line). Moreover, bi-2H10 appeared to block efficiently the process even at 2H10:N-MPER ratios below 1:3 (mol:mol), consistent with the involvement of peptide oligomers in the promotion of membrane fusion. Based on these data, we suggest that both 2H10-RKRF and bi-2H10 neutralize HIV-1 at the stage of lipid mixing.

\section{GP41FP-TM interaction with MPER bnAbs}

Although the $2 \mathrm{H} 10$ epitope overlaps with the 2F5 MPER epitope ${ }^{41}$, the $2 \mathrm{~F} 5$-bound peptide structure ${ }^{41}$, cannot be superimposed without major clashes with adjacent gp41 protomers. In contrast, $\mathrm{C} \alpha$ superposition of the structures of 10E8 and LN01 in complex with MPER peptides demonstrated possible binding to gp41FP-TM chain C-C (Fig. 3A and B). Furthermore, HCDR3 
of both 10E8 and LN01 could make additional hydrophobic contacts with adjacent FP in this binding mode. To confirm 10E8 and LN01 interaction, we performed immunoprecipitation of gp41FP-TM with both bnAbs, which confirmed their interaction in vitro (Fig. S2D). We next validated binding by bio-layer interferometry (BLI) using gp41FP-TM as analyte. This revealed $\mathrm{K}_{\mathrm{DS}}$ of $0,2 \mathrm{nM}$ for 10E8 and $34 \mathrm{nM}$ for LN01 (Fig. 3C and D). We conclude that bnAbs 10E8 and LN01 interact with gp41FP-TM with high affinity likely by inducing and stabilizing an asymmetric gp41 conformation similar to the one observed in complex with $2 \mathrm{H} 10$ as suggested by the structural modeling (Fig. 3 and B).

\section{Building a post fusion conformation by MD simulation}

In order to follow the final refolding of the membrane anchors we modeled the post fusion conformation employing MD simulation. Assuming that the final post-fusion conformation shows a straight symmetric rod-like structure we constructed a model of gp41 from the protomer composed of the straight helical chains N-B and C-B (Fig. S6A and B). This conformation is also present in the symmetric six-helix bundle structures containing either MPER ${ }^{35}$ or FPPR and MPER ${ }^{33}$ (Fig. S3). In this model, FP and TM do not interact tightly (Fig. S6B), which, however, does not explain the increased thermostability induced by FP and TM (Fig. S2A). 1- $\mu$ s MD simulation of this model (Fig. S6B) in solution, rearranges the membrane anchors such that they adopt a compact structure with trimeric FP interacting with adjacent TMs. Furthermore, the TMs kink at the conserved Gly positions 690 and 691, as observed previously ${ }^{42}$ (Fig. S6C). In order to recapitulate the stability of the model in the membrane, we performed an additional 1- $\mu$ s MD simulation of the model (Fig. S6C) in a bilayer resembling the HIV-1 lipid composition, which relaxed the TM to its straight conformation (Fig. 4A). The final structural model reveals tight packing of trimeric FP flexibly linked to HR1 by FPPR G525 to G527 (Fig. 4B). HR2-MPER and TMR form continuous helices with the TMRs packing against trimeric FP (Fig. 4A and C), which spans one monolayer (Fig. 4A). As conserved tryptophan residues within MPER have been previously implicated in fusion ${ }^{28,30}$, we analyzed their structural role in the post fusion model. This reveals that the indole ring of W666 is sandwiched between Leu669 and T536 and packs against L537. W670 makes a coiled-coil interaction with S534, while W672 is partially exposed and packs against L669 and T676. W678 binds into a hydrophobic pocket defined by I675, L679, I682 and adjacent FP/FPPR residues F522 and A526. W680 is partially exposed, but reaches into a pocket created by the flexible FPPR coil (Fig. S7). Thus, most of the tryptophan residues have structural roles in the post-fusion conformation, hence providing an explanation for their functional role in fusion ${ }^{28}$. The MPER epitopes recognized by 10E8 and LN01 are exposed in the post-fusion model, but antibody docking 
to this conformation produced major clashes, consistent with no expected binding to the final post fusion conformation.

\section{Structural transitions of gp41}

A number of Env SOSIP structures revealed the native conformation of gp41 (Fig. 5A and C) $9,43,44$. The gp41FP-TM crystal structure and the model of its post fusion conformation provide further insight into the path of conformational changes that native gp41 must undergo to adopt its final lowest energy state conformation. The first major conformational changes in gp41 that take place upon receptor binding are extension of HR1, FPPR and FP into a triple stranded coiled coil with flexible linkers between FPPR and FP that projects FP $\sim 115 \AA$ away from its starting position (Fig. 5D). Notably, such an early intermediate fusion conformation structure has been reported for influenza hemagglutinin (HA) ${ }^{45}$. This is likely followed by an extension and rearrangement of HR2 and MPER producing 11-15-nm long intermediates that connect the viral and cellular membranes ${ }^{20,46}$. Gp41 refolding into the six-helix bundle structure then produces flexibly linked asymmetric conformations of FPPR-FP and MPER-TMR anchored in the cellular and viral membranes, respectively, as indicated by the gp41FP-TM structure. This intermediate conformation may bring viral and cellular membranes into close proximity (Fig. 5E) or may act at the subsequent stage of hemifusion (Fig. 5F). Further refolding and interaction of FP-FPPR and MPER-TM will generate the stable post fusion conformation (Fig. 5G), a process that completes membrane fusion.

\section{Discussion}

Membrane fusion is an essential step of infection for enveloped viruses such as HIV-1, and requires extensive conformational rearrangements of the Env prefusion conformation ${ }^{7-9}$ into the final inactive post-fusion conformation ${ }^{2,3}$. The fusion model predicts that six-helix bundle formation apposes viral and cellular membranes with FP and TM inserted asymmetrically in the cellular membrane and the viral membrane, respectively ${ }^{21}$. Here, we show that gp41 containing its membrane anchors can adopt this predicted conformation, which is facilitated by flexible hinges present in FPPR and MPER, thus corroborating their essential roles in membrane fusion ${ }^{3,5,47}$. The asymmetric conformation of the membrane anchors suggest further that bundle formation occurs before pore formation as suggested previously ${ }^{26,27}$. The membrane-fusion model proposes further that final steps in fusion involves rearrangement and interaction of TM and FP ${ }^{21}$, which is confirmed by the MD-simulation model of the post-fusion conformation. Furthermore, the length of the rod-like post-fusion structure of $13 \mathrm{~nm}$ lacking the C-C loop is consistent with the gp41 structure lacking FP and $\mathrm{TM}^{48}$. 
FP is helical in the gp41FP-TM-2H10 complex and the MD-based post-fusion conformation, in agreement with NMR-based helical FP peptide models ${ }^{49,50}$, although $\beta$-strand structures of FP have been implicated in fusion as well ${ }^{51}$. In comparison, in native Env conformations, FP adopts multiple dynamic conformations that are recognized by broadly neutralizing antibodies $43,44,52,53$. In the post-fusion conformation, FP spans one monolayer of the membrane, in contrast to suggested amphipathic helix-like interaction of FP with the outer layer of the target cell membrane ${ }^{54,55}$.

Furthermore, the coiled-coil interactions within FP and with TM in the post fusion model explain the increased thermostability of gp41FP-TM compared to gp41 lacking FP and TM ${ }^{33}$. We propose that refolding of FP and TM can liberate additional free energy to catalyze final steps of fusion. Hence, replacement of TM by a phosphatidylinositol (PI) anchor inhibits membrane fusion 56,57 , akin to the GPI-anchored HA inhibition of influenza virus membrane fusion at the stage of hemifusion ${ }^{58}$.

Mutations in MPER and FPPR interfere with fusion $28,30,59,60$, and mutations in TM block fusion ${ }^{31}$ or reduce fusion efficiency ${ }^{32}$. Our structural model of the post-fusion conformation predicts that most of these mutations affect the final post-fusion conformation, in agreement with proposed interactions of FPPR and MPER, as well as FP and TM ${ }^{61,62}$, thereby corroborating their essential roles at late stages of membrane fusion.

Gp41FP-TM interaction with the 2H10 MPER-specific nanobody induces the asymmetric conformation of the membrane anchors. In order to confirm that $2 \mathrm{H} 10$ is, indeed, a neutralizing MPER-specific nanobody, we engineered increased 2H10 membrane binding, which improved breadth and potency of $2 \mathrm{H} 10$ neutralization, in agreement with enlarged potency by increasing membrane-binding of 10E8 ${ }^{63-65}$. This result, thus confirmed $2 \mathrm{H} 10$ as a modest anti-MPER neutralizing antibody that recognizes both its linear epitope and membrane ${ }^{34}$. Consistent with its neutralization capacity, $2 \mathrm{H} 10$ inhibits membrane fusion at the stage of lipid mixing like $2 \mathrm{~F} 5$ and other anti-MPER bnAbs ${ }^{40,66}$. Moreover, gp41FP-TM interacts with MPER bnAbs $10 \mathrm{E} 8{ }^{37}$ and LN01 ${ }^{42}$ in agreement with docking both structures onto the kinked chain C-C MPER epitope. Notably, the kink in the MPER peptide in complex with $10 \mathrm{E} 8{ }^{37}$ is similar to the chain C-C kink and present in MPER peptide NMR structures ${ }^{36,50}$. Furthermore, Ala mutations in the kink (671674) affect cell-cell fusion and lower virus infectivity ${ }^{67}$ corroborating the physiological relevance of the kinked conformation. We therefore propose that 10E8 and LN01 binding to gp41FP-TM induces similar asymmetry, as observed in the gp41FP-TM-2H10 structure by sampling the dynamic states of the membrane anchors. 
Our data, thus, indicate that MPER antibodies can act all along the gp41 refolding pathway from blocking initial conformations of close to native Env ${ }^{68-70}$ up to a late fusion intermediate state that has already pulled viral and cellular membranes into close apposition. This thus, opens a long

312 temporal window of action for MPER bnAbs consistent with the findings that the half-life of neutralization of MPER bnAbs is up to 30 minutes post virus exposure to target cells ${ }^{71,72}$.

\section{Materials and Methods}

\section{Cell Lines}

TZM-bl cells were obtained from NIH-AIDS Research and Reference Reagent Program (ARRRP) and used for neutralization assays. TZM-bl cells were maintained in Dulbecco's modified Eagle's medium supplemented with $10 \%$ fetal bovine serum, 100 units of Penicillin and $0.1 \mathrm{mg} / \mathrm{ml} \mathrm{of}$ Streptomycin while TZM-bl expressing the Fc $\gamma$ RI cells were maintained in Dulbecco's modified Eagle's medium supplemented with 10\% fetal bovine serum, 0.025M Hepes, $50 \mu \mathrm{g} / \mathrm{ml}$ of Gentamicin, $20 \mu \mathrm{g} / \mathrm{ml}$ of Blasticidin.

\section{HIV-1 Primary Viruses}

Env-pseudotyped viruses were prepared by co-transfection of HEK 293-T cells with plasmids encoding the respective env genes and the luciferase reporter HIV vector pNLluc-AM as described 73. A full list of Env pseudotyped viruses generated with corresponding gene bank entry, subtype and Tier information is provided in Table S2.

\section{GP41 expression and purification}

DNA fragments encoding HIV-1 Env glycoprotein amino acids 512 to 581 (N-terminal chain, chain $\mathrm{N}$ ) and residues 629 to 715 (C-terminal chain, chain C) were cloned into vectors pETM20 and pETM11 (PEPcore facility-EMBL), respectively. Both constructs contain an N-terminal Flag-tag (DDDDK sequence) and chain $\mathrm{C}$ contains additional two C-terminal arginine residues (Fig. S1A). Proteins were expressed separately in E. coli strain C41(DE3)(Lucigen). Bacteria were grown at $37^{\circ} \mathrm{C}$ to an $\mathrm{OD}_{600 \mathrm{~nm}}$ of 0,9 . Cultures were induced with $1 \mathrm{mM}$ IPTG at $37^{\circ} \mathrm{C}$ for $3 \mathrm{~h}$ for gp 41 chain $\mathrm{N}$ and at $25^{\circ} \mathrm{C}$ for $20 \mathrm{~h}$ for gp41 chain $\mathrm{C}$. Cells were lysed by sonication in buffer A containing 20 
$\mathrm{mM}$ Tris $\mathrm{pH}$ 8, $100 \mathrm{mM} \mathrm{NaCl}$ and 1\% CHAPS (3-[(3-cholamidopropyl) diméthylammonio]-1propanesulfonate (Euromedex). The supernatant was cleared by centrifugation at $53000 \mathrm{~g}$ for 30 min. Gp41 chain $\mathrm{N}$ supernatant was loaded on a $\mathrm{Ni}^{2}$-sepharose column, washed successively with Buffer A containing $1 \mathrm{M} \mathrm{NaCl}$ and $1 \mathrm{M} \mathrm{KCl}$, then Buffer A containing $50 \mathrm{mM}$ imidazole. Gp41 chain $\mathrm{N}$ was eluted in Buffer A containing $500 \mathrm{mM}$ imidazole. Gp41 chain $\mathrm{C}$ was purified employing the same protocol as for gp41 chain N. Gp41 chain N was subsequently cleaved with TEV (Tobacco Etch Virus) protease for $2 \mathrm{~h}$ at $20^{\circ} \mathrm{C}$ and then overnight at $4{ }^{\circ} \mathrm{C}$. After buffer exchange with a mono Q column using buffer $\mathrm{B}$ (Buffer $\mathrm{A}$ with $0,5 \mathrm{M} \mathrm{NaCl}$ ), uncleaved material and cleaved His-tags were removed by a second $\mathrm{Ni}^{2+}$-sepharose column in buffer $\mathrm{A}$. TEV-cleaved gp41 chain $\mathrm{C}$ and chain $\mathrm{N}$ were then mixed in a molar ratio 4:1 and incubated overnight. To remove the excess of gp41 chain $\mathrm{C}$, the gp41 complex was loaded on a $3 \mathrm{rd} \mathrm{Ni}^{2+}$-sepharose column in buffer $\mathrm{A}$, washed with buffer A containing $50 \mathrm{mM}$ imidazole and eluted with buffer A containing $500 \mathrm{mM}$ imidazole. Subsequently the gp 41 chain N TrxA-His-tag was removed by TEV digestion for $2 \mathrm{~h}$ at $20^{\circ} \mathrm{C}$ and overnight at $4{ }^{\circ} \mathrm{C}$. After buffer exchange with a mono Q column in buffer B uncleaved complex and the TrxA-His-tag fusion were removed by a 4 th $\mathrm{Ni}^{2+}$-sepharose column. The final gp41FP-TM complex was concentrated and loaded onto a Superdex 200 size exclusion column (SEC) in buffer $\mathrm{C}$ containing $20 \mathrm{mM}$ Tris $\mathrm{pH} 8,0,100 \mathrm{mM} \mathrm{NaCl}$ and $1 \% \mathrm{n}$-octyl $\beta$-D-glucopyranoside (Anatrace).

\section{Nanobody $2 \mathrm{H} 10$ expression}

2H10 encoding DNA was cloned into the vector pAX51 ${ }^{34}$ and expressed in the E. coli BL21(DE3) strain (Invitrogen). Bacteria were grown at $37^{\circ} \mathrm{C}$ to an $\mathrm{OD}_{600 \mathrm{~nm}}$ of 0,7 and induced with $1 \mathrm{mM}$ IPTG at $20^{\circ} \mathrm{C}$ for $20 \mathrm{~h}$. After harvesting by centrifugation, bacteria were resuspended in lysis buffer containing $20 \mathrm{mM}$ Hepes $\mathrm{pH} 7,5$ and $100 \mathrm{mM} \mathrm{NaCl}$. Bacteria were lysed by sonication and centrifuged at $48000 \mathrm{~g}$ for $30 \mathrm{~min}$. Cleared supernatant was loaded on Protein A sepharose column, washed with lysis buffer and eluted with $0,1 \mathrm{M}$ glycine $\mathrm{pH}$ 2,9. Eluted fractions were immediately mixed with $1 / 5$ volume of $1 \mathrm{M}$ Tris $\mathrm{pH} 9,0.2 \mathrm{H} 10$ was then further purified by SEC on a superdex 75 column in PBS buffer. Mutants of 2H10, 2H10-F (S100d) and 2H10-RKRF (S27R, S30K, S74R and S100d) were synthesized (Biomatik) and purified as described for the wild type. The 2H10 bihead was purified as described ${ }^{34}$.

\section{Circular dichroism}

CD measurements were performed using a JASCO Spectropolarimeter equipped with a thermoelectric temperature controller. Spectra of gp41-TM were recorded at $20^{\circ} \mathrm{C}$ in $1 \mathrm{~nm}$ steps from 190 to $260 \mathrm{~nm}$ in a buffer containing PBS supplemented with $1 \%$ n-octyl $\beta$-D- 
glucopyranoside. For thermal denaturation experiments, the ellipticity was recorded at $222 \mathrm{~nm}$ with $1^{\circ} \mathrm{C}$ steps from $20^{\circ}$ to $95^{\circ} \mathrm{C}$ with an increment of $80^{\circ} \mathrm{C} \mathrm{h}^{-1}$, and an averaging time of $30 \mathrm{~s} / \mathrm{step}$. For data analysis, raw ellipticity values recorded at $222 \mathrm{~nm}$ were converted to mean residue ellipticity.

\section{Isothermal Titration Calorimetry (ITC)}

The stoichiometry and binding constants of 2H10 binding to gp41 FP-TM was measured by ITC200 (MicroCal Inc.). All samples used in the ITC experiments were purified by SEC in a buffer containing $20 \mathrm{mM}$ Tris $\mathrm{pH} 8.0,100 \mathrm{mM} \mathrm{NaCl}$ and $1 \%$ n-octyl $\beta$-D glucopyranoside and used without further concentration. Samples and were equilibrated at $25{ }^{\circ} \mathrm{C}$ before the start of the experiment. The ITC measurements were performed at $25^{\circ} \mathrm{C}$ by making $202-\mu 1$ injections of 267 $\mu \mathrm{M} 2 \mathrm{H} 10$ to $0.2 \mathrm{ml}$ of $19.5 \mu \mathrm{M}$ gp41FP-TM. Curve fitting was performed with MicroCal Origin software. Three experiments were performed, with an average stoichiometry $\mathrm{N}=1.1+/-0.22 \mathrm{H} 10$ binds to gp41FP-TM with a KD of $2.1 \mu \mathrm{M}+/-0.9$.

\section{Bio-layer Interferometry Binding Analysis}

Binding measurements between antibodies (10E8 IgG, LN01 IgG and 2H10) were carried out on an Octet Red instrument (ForteBio). For the determination of the binding between antibodies and gp41FP-TM, 10E8 IgG or LN01 IgG or 2H10 were labelled with biotin (EZ-Link NHS-PEG4Biotin) and bound to Streptavidin (SA) biosensors (ForteBio). The biosensors loaded with the antibodies were equilibrated in the kinetic buffer $(20 \mathrm{mM}$ Tris pH 8.0, $100 \mathrm{mM} \mathrm{NaCl}$ and $1 \% \mathrm{n}$ octyl $\beta$-D glucopyranoside) for $200-500 \mathrm{sec}$ prior to measuring association with different concentrations of gp41FP- for $100-200$ seconds at $25^{\circ} \mathrm{C}$. Data were analyzed using the ForteBio analysis software version 11.1.0.25 (ForteBio). For 10E8 the kinetic parameters were calculated using a global fit 1:1 model and 2:1 model. For the determination of the binding of LN01 IgG and $2 \mathrm{H} 10$, KDs were estimated by steady state analysis. All bio-layer interferometry experiments were conducted at least three times.

\section{Immunoprecipitation of gp41FP-TM by bnAbs 10E8 and LN01}

$220 \mu \mathrm{g}$ of Gp41FP-TM were incubated alone or with $50 \mu \mathrm{g}$ of 10E8 or LN01 antibodies for $10 \mathrm{~h}$ at $20^{\circ} \mathrm{C}$ in buffer $\mathrm{C}$. The complex was loaded on Protein A sepharose affinity resin and incubated for 1h. The resin was subsequently washed 3 times with buffer C and eluted with SDS gel loading buffer and boiling at $95^{\circ} \mathrm{C}$ for $5 \mathrm{~min}$. Samples were separated on a $15 \%$ SDS-PAGE and stained with Coomassie brilliant blue. 


\section{Neutralization assay}

The neutralization activity of the $2 \mathrm{H} 10$ variants and mAbs was evaluated using TZM-bl cells and Env pseudotyped viruses as described ${ }^{73}$. Briefly, serial dilutions of inhibitor were prepared in cell culture medium (DMEM with 10\% heat-inactivated FBS, $100 \mathrm{U} / \mathrm{ml}$ penicillin and $100 \mu \mathrm{g} / \mathrm{ml}$ streptomycin (all from Gibco)) and added at a 1:1 volume ratio to pseudovirus suspension in 384 well plates (aiming for 500'000-5'000'000 relative light units (RLU) per well in the absence of inhibitors). After one-hour incubation at $37^{\circ} \mathrm{C}, 30 \mu \mathrm{l}$ of virus-inhibitor mixture was transferred to TZM-bl cells in 384 well plates (6000 cells/well in $30 \mu 1$ cell culture medium supplemented with $20 \mu \mathrm{g} / \mathrm{ml}$ DEAE-Dextran seeded the previous day). The plates were further incubated for 48 hours at $37^{\circ} \mathrm{C}$ before readout of luciferase reporter gene expression on a Perkin Elmer EnVision Multilabel Reader using the Bright-Glo Luciferase Assay System (Promega).

The inhibitor concentration (referring to the mix with cells, virus and inhibitor) causing 50\% reduction in luciferase signal with respect to a reference well without inhibitor (inhibitory concentration IC50) was calculated by fitting a non-linear regression curve (variable slope) to data from two independent experiments using Prism (GraphPad Software). If 50\% inhibition was not achieved at the highest inhibitor concentration tested, a greater than value was recorded. To control for unspecific effects all inhibitors were tested for activity against MuLV envelope pseudotyped virus.

\section{Fusion assay.}

The peptides used in the fusion inhibition experiments, NEQELLELDKWASLW NWFNITNWLWYIK (N-MPER) and $K K K$-NWFDITNWLWYIKLFIMIVGGLV-KK (C-MPER), were synthesized in C-terminal carboxamide form by solid-phase methods using Fmoc chemistry, purified by reverse phase HPLC, and characterized by matrix-assisted time-of-flight (MALDITOF) mass spectrometry (purity $>95 \%$ ). Peptides were routinely dissolved in dimethylsulfoxide (DMSO, spectroscopy grade) and their concentration determined by the Biscinchoninic Acid microassay (Pierce, Rockford, IL, USA).

Large unilamellar vesicles (LUV) were prepared following the extrusion method of Hope et al. ${ }^{74}$. 1-palmitoyl-2-oleoylphosphatidylcholine (POPC) and cholesterol (Chol) (Avanti Polar Lipids, Birmingham, AL, USA) were mixed in chloroform at a 2:1 mol:mol ratio and dried under a $\mathrm{N}_{2}$ stream. Traces of organic solvent were removed by vacuum pumping. Subsequently, the dried lipid films were dispersed in $5 \mathrm{mM}$ Hepes and $100 \mathrm{mM} \mathrm{NaCl}$ (pH 7.4) buffer, and subjected to 10 freezethaw cycles prior to extrusion 10 times through 2 stacked polycarbonate membranes (Nuclepore, Inc., Pleasanton, CA, USA). Lipid mixing with fusion-committed vesicles was monitored based on 
the resonance energy transfer assay described by Struck et al. ${ }^{75}$, with the modifications introduced by Apellaniz et al. ${ }^{40}$. The assay is based on the dilution of co-mixed N-(7-nitro-benz-2-oxa-1,3diazol-4-yl)phosphatidylethanolamine (N-NBD-PE) and N-(lissamine Rhodamine B sulfonyl)phosphatidylethanolamine (N-Rh-PE) (Molecular Probes, Eugene, OR, USA), whereby dilution due to membrane mixing results in increased N-NBD-PE fluorescence. Vesicles containing $0.6 \mathrm{~mol} \%$ of each probe (target vesicles) were added at 1:9 ratio to unlabeled vesicles (MPER peptide-primed vesicles). The final lipid concentration in the mixture was $100 \mu \mathrm{M}$. The increase in NBD emission upon mixing of target-labeled and primed-unlabeled lipid bilayers was monitored at $530 \mathrm{~nm}$ with the excitation wavelength set at $465 \mathrm{~nm}$. A cutoff filter at $515 \mathrm{~nm}$ was used between the sample and the emission monochromator to avoid scattering interferences. The fluorescence scale was calibrated such that the zero level corresponded to the initial residual fluorescence of the labeled vesicles and the $100 \%$ value to complete mixing of all the lipids in the system (i.e., the fluorescence intensity of vesicles containing $0.06 \mathrm{~mol} \%$ of each probe). Fusion inhibition was performed with bi-2H10, 2H10-RKRF and 2F5 Fabs at concentrations of $10 \mu \mathrm{g} / \mathrm{ml}$ and $20 \mu \mathrm{g} / \mathrm{ml}$ as indicated.

\section{Crystallization, data collection and structure determination}

For crystallization, $1 \mathrm{mg}$ of gp41FP-TM was mixed with $1.5 \mathrm{mg}$ of $2 \mathrm{H} 10$. The complex was purified by SEC on a Superdex 200 column in a buffer containing $100 \mathrm{mM} \mathrm{NaCl,} 20 \mathrm{mM}$ Tris pH 8,0 and $1 \% \mathrm{n}$-octyl $\beta$-D-glucopyranoside and concentrated to $7-10 \mathrm{mg} / \mathrm{ml}$. Crystal screening was performed at the EMBL High Throughput Crystallization Laboratory (HTX lab, Grenoble) in 96-well sitting drop vapor diffusion plates (Greiner). Following manual refinement of crystallization conditions, crystals of gp41FP-TM in complex with $2 \mathrm{H} 10$ were grown by mixing $1 \mu \mathrm{l}$ of protein with $1 \mu \mathrm{l}$ of reservoir buffer containing $0,1 \mathrm{M}$ sodium citrate $\mathrm{pH} 6,0,0,2 \mathrm{M}$ ammonium sulfate, 20\% polyethylene glycol 2000 and $0,1 \mathrm{M} \mathrm{NaCl}$ at $20^{\circ} \mathrm{C}(293 \mathrm{~K})$ in hanging drop vapor diffusion plates. Before data collection, crystals were flash frozen at $100 \mathrm{~K}$ in reservoir solution supplemented with $1 \%$ n-octyl $\beta$-D-glucopyranoside and $25 \%$ ethylene glycol for cryo-protection.

Data were collected on the ESRF beamline ID30b at a wavelength of $0.9730 \AA$. Data were processed with the program XDS ${ }^{76}$. The data from two crystals were merged with Aimless ${ }^{77}$. The data set displayed strong anisotropy in its diffraction limits and was submitted to the STARANISO Web server ${ }^{78}$. The merged STARANISO protocol produced a best-resolution limit of $3.28 \AA$ and a worst-resolution limit of $4.74 \AA$ at a cutoff level of 1.2 for the local $\mathrm{I}_{\text {mean }} / \sigma\left(\mathrm{I}_{\text {mean }}\right)$ (note that STARANISO does not employ ellipsoidal truncations coincident with the crystal axes). The gp41FP-TM-2H10 crystals belong to space group C $2222_{1}$ and the structure was solved by 
molecular replacement using the program Phaser ${ }^{79}$ and pdb entries 1env and $4 \mathrm{~b} 50$. The model was rebuilt using $\mathrm{COOT}^{80}$ and refined using Phenix ${ }^{81}$. Data up to $3.28 \AA$ were initially used for model building but were finally truncated to $3.8 \AA$. Statistics for data reduction and structure refinement are presented in Table S1.

One copy of gp41FP-TM in complex with $2 \mathrm{H} 10$ are present in the asymmetric unit. Numbering of the nanobody $2 \mathrm{H} 10$ was performed according to Kabat. The gp41FP-TM-2H10 complex was refined to $3.8 \AA$ data with an $\mathrm{R} / \mathrm{R}$ free of 26.7 / $31.1 \% .99 .6 \%$ of the residues are within the most favored and allowed regions of a Ramachandran plot ${ }^{77}$. Some of the crystallographic software used were compiled by SBGrid ${ }^{82}$. Atomic coordinates and structure factors of the reported crystal structures have been deposited in the Protein Data Bank (https://www.rcsb.org; PDB: 7AEJ.

\section{Figure Generation}

Molecular graphics figures were generated with PyMOL (W. Delano; The PyMOL Molecular Graphics System, Version 1.8 Schrödinger, LLC, http://www.pymol.org).

\section{Molecular Dynamics (MD) simulation}

Molecular assays. Starting from the crystal structure determined herein, we built two molecular assays based (i) on the structure of the entire Gp41FP-TM/2H10 complex, and (ii) based on a gp41 model generated by a three-fold symmetrization of the straight helical chains N-B and C-B. Electron density for FP and TM is partially absent in the crystal structure and the missing parts have been built as helical extensions; FP from residue 512 to 518 and TM from residues 700 to 709 based on TM structures (6SNE and 6B3U). All residues were taken in their standard protonation state. The first assay included a fully hydrated membrane composed of 190 cholesterol, 40 1-palmitoyl2-oleoyl-glycero-3-phosphocholine (POPC), 88 1-palmitoyl-2-oleoyl-sn-glycero-3-phosphoethanolamine (POPE), 36 1-palmitoyl-2-oleoyl-sn-glycero-3-phospho-L-serine (POPS) and $56 \mathrm{~N}$ stearoyl sphingomyelin, present in the HIV-1 lipid envelope ${ }^{83}$, using the CHARMM-GUI interface 84,85 . The resulting molecular assembly consisted of about 178,000 atoms in a rhomboidal cell of $106 \times 106 \times 169 \AA^{3}$. The second computational assay featured a water bath of $91 \times 91 \times 114 \AA^{3}$, representing a total of nearly 95,700 atoms. Both assays were electrically neutral, with a $\mathrm{NaCl}$ concentration set to $150 \mathrm{mM}$.

Molecular Dynamics. All simulations were performed using the NAMD 2.14 program ${ }^{86}$. Proteins, cholesterol, lipids and ions were described using the CHARMM forcefield ${ }^{87-89}$ and the TIP3P model ${ }^{90}$ was used for water. MD trajectories were generated in the isobaric-isothermal ensemble at a temperature of $300 \mathrm{~K}$ and a pressure of $1 \mathrm{~atm}$. Pressure and temperature were kept constant 
513 using the Langevin thermostat and the Langevin piston method ${ }^{91}$, respectively. Long-range

514 electrostatic interactions were evaluated by the particle-mesh Ewald (PME) algorithm ${ }^{92}$. Hydrogen

1. Kielian, M. \& Rey, F.A. Virus membrane-fusion proteins: more than one way to make a hairpin. Nat Rev Microbiol 4, 67-76 (2006).

2. Weissenhorn, W., Hinz, A. \& Gaudin, Y. Virus membrane fusion. FEBS Lett 581, 2150-5 (2007).

3. Harrison, S.C. Viral membrane fusion. Nat Struct Mol Biol 15, 690-698 (2008).

4. Baquero, E., Albertini, A.A. \& Gaudin, Y. Recent mechanistic and structural insights on class III viral fusion glycoproteins. Curr Opin Struct Biol 33, 52-60 (2015).

5. Chen, B. Molecular Mechanism of HIV-1 Entry. Trends Microbiol (2019).

6. Wang, Q., Finzi, A. \& Sodroski, J. The Conformational States of the HIV-1 Envelope Glycoproteins. Trends Microbiol 28, 655-667 (2020).

7. Julien, J.P. et al. Crystal Structure of a Soluble Cleaved HIV-1 Envelope Trimer. Science 342, 1477-83 (2013).

8. Lyumkis, D. et al. Cryo-EM Structure of a Fully Glycosylated Soluble Cleaved HIV-1 Envelope Trimer. Science 342, 1484-90 (2013).

9. Pancera, M. et al. Structure and immune recognition of trimeric pre-fusion HIV-1 Env. Nature 514, 455-61 (2014).

10. Kwon, Y.D. et al. Crystal structure, conformational fixation and entry-related interactions of mature ligand-free HIV-1 Env. Nat Struct Mol Biol 22, 522-31 (2015).

11. Stewart-Jones, G.B. et al. Trimeric HIV-1-Env Structures Define Glycan Shields from Clades A, B, and G. Cell 165, 813-26 (2016).

12. Ward, A.B. \& Wilson, I.A. The HIV-1 envelope glycoprotein structure: nailing down a moving target. Immunol Rev 275, 21-32 (2017).

13. Liu, J., Bartesaghi, A., Borgnia, M.J., Sapiro, G. \& Subramaniam, S. Molecular architecture of native HIV-1 gp120 trimers. Nature 455, 109-13 (2008). 
14. Ozorowski, G. et al. Open and closed structures reveal allostery and pliability in the HIV1 envelope spike. Nature 547, 360-363 (2017).

15. Wang, H., Barnes, C.O., Yang, Z., Nussenzweig, M.C. \& Bjorkman, P.J. Partially Open HIV-1 Envelope Structures Exhibit Conformational Changes Relevant for Coreceptor Binding and Fusion. Cell Host Microbe 24, 579-592 e4 (2018).

16. Shaik, M.M. et al. Structural basis of coreceptor recognition by HIV-1 envelope spike. Nature 565, 318-323 (2019).

17. Dobrowsky, T.M., Zhou, Y., Sun, S.X., Siliciano, R.F. \& Wirtz, D. Monitoring early fusion dynamics of human immunodeficiency virus type 1 at single-molecule resolution. $J$ Virol 82, 7022-33 (2008).

18. Chang, M.I., Panorchan, P., Dobrowsky, T.M., Tseng, Y. \& Wirtz, D. Single-molecule analysis of human immunodeficiency virus type 1 gp120-receptor interactions in living cells. J Virol 79, 14748-55 (2005).

19. Frey, G. et al. A fusion-intermediate state of HIV-1 gp41 targeted by broadly neutralizing antibodies. Proc Natl Acad Sci U S A 105, 3739-44 (2008).

20. Lai, R.P. et al. A fusion intermediate gp41 immunogen elicits neutralizing antibodies to HIV-1. J Biol Chem 289, 29912-26 (2014).

21. Weissenhorn, W. et al. Structural basis for membrane fusion by enveloped viruses. Mol Membr Biol 16, 3-9 (1999).

22. Weissenhorn, W., Dessen, A., Harrison, S.C., Skehel, J.J. \& Wiley, D.C. Atomic structure of the ectodomain from HIV-1 gp41. Nature 387, 426-30 (1997).

23. Chan, D.C., Fass, D., Berger, J.M. \& Kim, P.S. Core structure of gp41 from the HIV envelope glycoprotein. Cell 89, 263-73 (1997).

24. Caffrey, M. et al. Three-dimensional solution structure of the $44 \mathrm{kDa}$ ectodomain of SIV gp41. Embo J 17, 4572-84 (1998).

25. Chernomordik, L.V. \& Kozlov, M.M. Membrane Hemifusion: Crossing a Chasm in Two Leaps. Cell 123, 375-382 (2005).

26. Melikyan, G.B. et al. Evidence that the transition of HIV-1 gp41 into a six-helix bundle, not the bundle configuration, induces membrane fusion. J Cell Biol 151, 413-23 (2000).

27. Markosyan, R.M., Cohen, F.S. \& Melikyan, G.B. HIV-1 envelope proteins complete their folding into six-helix bundles immediately after fusion pore formation. Mol Biol Cell 14, 926-38 (2003).

28. Salzwedel, K., West, J.T. \& Hunter, E. A conserved tryptophan-rich motif in the membrane-proximal region of the human immunodeficiency virus type $1 \mathrm{gp} 41$ ectodomain is important for Env-mediated fusion and virus infectivity. J Virol 73, 246980 (1999).

29. Peisajovich, S.G., Epand, R.F., Pritsker, M., Shai, Y. \& Epand, R.M. The polar region consecutive to the HIV fusion peptide participates in membrane fusion. Biochemistry 39, 1826-33 (2000).

30. Bellamy-McIntyre, A.K. et al. Functional links between the fusion peptide-proximal polar segment and membrane-proximal region of human immunodeficiency virus gp41 in distinct phases of membrane fusion. J Biol Chem 282, 23104-16 (2007).

31. Shang, L., Yue, L. \& Hunter, E. Role of the membrane-spanning domain of human immunodeficiency virus type 1 envelope glycoprotein in cell-cell fusion and virus infection. J Virol 82, 5417-28 (2008).

32. Long, Y., Meng, F., Kondo, N., Iwamoto, A. \& Matsuda, Z. Conserved arginine residue in the membrane-spanning domain of HIV-1 gp41 is required for efficient membrane fusion. Protein Cell 2, 369-76 (2011).

33. Buzon, V. et al. Crystal structure of HIV-1 gp41 including both fusion peptide and membrane proximal external regions. PLoS Pathog 6, e1000880 (2010). 
34. Lutje Hulsik, D. et al. A gp41 MPER-specific llama VHH requires a hydrophobic CDR3 for neutralization but not for antigen recognition. PLoS Pathog 9, e1003202 (2013).

35. Shi, W. et al. Structural characterization of HIV gp41 with the membrane-proximal external region. J Biol Chem 285, 24290-8 (2010).

36. Sun, Z.Y. et al. HIV-1 broadly neutralizing antibody extracts its epitope from a kinked gp41 ectodomain region on the viral membrane. Immunity 28, 52-63 (2008).

37. Huang, J. et al. Broad and potent neutralization of HIV-1 by a gp41-specific human antibody. Nature 491, 406-12 (2012).

38. Serrano, S. et al. Structure-Related Roles for the Conservation of the HIV-1 Fusion Peptide Sequence Revealed by Nuclear Magnetic Resonance. Biochemistry 56, 5503-5511 (2017).

39. Cerutti, N., Loredo-Varela, J.L., Caillat, C. \& Weissenhorn, W. Antigp41 membrane proximal external region antibodies and the art of using the membrane for neutralization. (2017).

40. Apellaniz, B. et al. Cholesterol-dependent membrane fusion induced by the gp41 membrane-proximal external region-transmembrane domain connection suggests a mechanism for broad HIV-1 neutralization. J Virol 88, 13367-77 (2014).

41. Ofek, G. et al. Structure and mechanistic analysis of the anti-human immunodeficiency virus type 1 antibody 2F5 in complex with its gp41 epitope. J Virol 78, 10724-37 (2004).

42. Pinto, D. et al. Structural Basis for Broad HIV-1 Neutralization by the MPER-Specific Human Broadly Neutralizing Antibody LN01. Cell Host Microbe 26, 623-637 e8 (2019).

43. Yuan, M. et al. Conformational Plasticity in the HIV-1 Fusion Peptide Facilitates Recognition by Broadly Neutralizing Antibodies. Cell Host Microbe 25, 873-883 e5 (2019).

44. Kumar, S. et al. Capturing the inherent structural dynamics of the HIV-1 envelope glycoprotein fusion peptide. Nat Commun 10, 763 (2019).

45. Benton, D.J., Gamblin, S.J., Rosenthal, P.B. \& Skehel, J.J. Structural transitions in influenza haemagglutinin at membrane fusion $\mathrm{pH}$. Nature 583, 150-153 (2020).

46. Ladinsky, M.S. et al. Electron tomography visualization of HIV-1 fusion with target cells using fusion inhibitors to trap the pre-hairpin intermediate. Elife 9(2020).

47. Blumenthal, R., Durell, S. \& Viard, M. HIV entry and envelope glycoprotein-mediated fusion. J Biol Chem 287, 40841-9 (2012).

48. Weissenhorn, W. et al. The ectodomain of HIV-1 env subunit gp41 forms a soluble, alphahelical, rod-like oligomer in the absence of gp120 and the N-terminal fusion peptide. EMBO J 15, 1507-14 (1996).

49. Li, Y. \& Tamm, L.K. Structure and Plasticity of the Human Immunodeficiency Virus gp41 Fusion Domain in Lipid Micelles and Bilayers. Biophys J. 93, 876-885 (2007).

50. Serrano, S. et al. Structure and immunogenicity of a peptide vaccine, including the complete HIV-1 gp41 2F5 epitope: implications for antibody recognition mechanism and immunogen design. J Biol Chem 289, 6565-80 (2014).

51. Apellaniz, B., Huarte, N., Largo, E. \& Nieva, J.L. The three lives of viral fusion peptides. Chem Phys Lipids 181, 40-55 (2014).

52. Blattner, C. et al. Structural delineation of a quaternary, cleavage-dependent epitope at the gp41-gp120 interface on intact HIV-1 Env trimers. Immunity 40, 669-80 (2014).

53. Kong, R. et al. Fusion peptide of HIV-1 as a site of vulnerability to neutralizing antibody. Science 352, 828-33 (2016).

54. Han, X., Bushweller, J.H., Cafiso, D.S. \& Tamm, L.K. Membrane structure and fusiontriggering conformational change of the fusion domain from influenza hemagglutinin. Nat Struct Biol 8, 715-20 (2001). 
55. Lai, A.L., Moorthy, A.E., Li, Y. \& Tamm, L.K. Fusion activity of HIV gp41 fusion domain is related to its secondary structure and depth of membrane insertion in a cholesterol-dependent fashion. J Mol Biol 418, 3-15 (2012).

56. Weiss, C.D. \& White, J.M. Characterization of stable Chinese hamster ovary cells expressing wild-type, secreted, and glycosylphosphatidylinositol-anchored human immunodeficiency virus type 1 envelope glycoprotein. J Virol 67, 7060-6 (1993).

57. Salzwedel, K., Johnston, P.B., Roberts, S.J., Dubay, J.W. \& Hunter, E. Expression and characterization of glycophospholipid-anchored human immunodeficiency virus type 1 envelope glycoproteins. J Virol 67, 5279-88. (1993).

58. Kemble, G.W., Danieli, T. \& White, J.M. Lipid-anchored influenza hemagglutinin promotes hemifusion, not complete fusion. Cell 76, 383-391 (1994).

59. Munoz-Barroso, I., Salzwedel, K., Hunter, E. \& Blumenthal, R. Role of the membraneproximal domain in the initial stages of human immunodeficiency virus type 1 envelope glycoprotein-mediated membrane fusion. J Virol 73, 6089-92 (1999).

60. Narasimhulu, V.G.S. et al. Distinct functions for the membrane-proximal ectodomain region (MPER) of HIV-1 gp41 in cell-free and cell-cell viral transmission and cell-cell fusion. J Biol Chem 293, 6099-6120 (2018).

61. Louis, J.M. et al. Insights into the Conformation of the Membrane Proximal Regions Critical to the Trimerization of the HIV-1 gp41 Ectodomain Bound to Dodecyl Phosphocholine Micelles. PLoS One 11, e0160597 (2016).

62. Reuven, E.M. et al. HIV-1 gp41 transmembrane domain interacts with the fusion peptide: implication in lipid mixing and inhibition of virus-cell fusion. Biochemistry 51, 2867-78 (2012).

63. Chen, J. et al. Mechanism of HIV-1 neutralization by antibodies targeting a membraneproximal region of gp41. J Virol 88, 1249-58 (2014).

64. Kwon, Y.D. et al. Surface-Matrix Screening Identifies Semi-specific Interactions that Improve Potency of a Near Pan-reactive HIV-1-Neutralizing Antibody. Cell Rep 22, 17981809 (2018).

65. Rujas, E. et al. Functional Optimization of Broadly Neutralizing Hiv-1 Antibody 10e8 by Promoting Membrane Interactions. J Virol (2018).

66. Huarte, N. et al. The broadly neutralizing anti-human immunodeficiency virus type 1 4E10 monoclonal antibody is better adapted to membrane-bound epitope recognition and blocking than 2F5. J Virol 82, 8986-96 (2008).

67. Sun, Z.Y. et al. Disruption of helix-capping residues 671 and 674 reveals a role in HIV-1 entry for a specialized hinge segment of the membrane proximal external region of gp41. $J$ Mol Biol 426, 1095-108 (2014).

68. Lee, J.H., Ozorowski, G. \& Ward, A.B. Cryo-EM structure of a native, fully glycosylated, cleaved HIV-1 envelope trimer. Science 351, 1043-8 (2016).

69. Rantalainen, K. et al. HIV-1 Envelope and MPER Antibody Structures in Lipid Assemblies. Cell Rep 31, 107583 (2020).

70. Carravilla, P. et al. Molecular recognition of the native HIV-1 MPER revealed by STED microscopy of single virions. Nat Commun 10, 78 (2019).

71. Shen, X. et al. Prolonged exposure of the HIV-1 gp41 membrane proximal region with L669S substitution. Proc Natl Acad Sci U S A 107, 5972-7 (2010).

72. Williams, L.D. et al. Potent and broad HIV-neutralizing antibodies in memory B cells and plasma. Sci Immunol 2(2017).

73. Rusert, P. et al. Divergent effects of cell environment on HIV entry inhibitor activity. AIDS 23, 1319-27 (2009). 
74. Hope, M.J., Bally, M.B., Webb, G. \& Cullis, P.R. Production of large unilamellar vesicles by a rapid extrusion procedure: characterization of size distribution, trapped volume and ability to maintain a membrane potential. Biochim Biophys Acta 812, 55-65 (1985).

75. Struck, D.K., Hoekstra, D. \& Pagano, R.E. Use of resonance energy transfer to monitor membrane fusion. Biochemistry 20, 4093-9 (1981).

76. Kabsch, W. Integration, scaling, space-group assignment and post-refinement. Acta Crystallogr D Biol Crystallogr 66, 133-44 (2010).

77. Evans, P.R. \& Murshudov, G.N. How good are my data and what is the resolution? Acta Crystallogr D Biol Crystallogr 69, 1204-14 (2013).

78. Tickle, I.J. et al. STARANISO. (http://staraniso.globalphasing.org/cgi-bin/staraniso.cgi). Cambridge, United Kingdom: Global Phasing Ltd. (2018).

79. McCoy, A.J. et al. Phaser crystallographic software. J Appl Crystallogr 40, 658-674 (2007).

80. Emsley, P., Lohkamp, B., Scott, W.G. \& Cowtan, K. Features and development of Coot. Acta Crystallogr D Biol Crystallogr 66, 486-501 (2010).

81. Adams, P.D. et al. PHENIX: a comprehensive Python-based system for macromolecular structure solution. Acta Crystallogr D Biol Crystallogr 66, 213-21 (2010).

82. Morin, A. et al. Collaboration gets the most out of software. Elife 2:e01456(2013).

83. Brugger, B. et al. The HIV lipidome: a raft with an unusual composition. Proc Natl Acad Sci U S A 103, 2641-6 (2006).

84. Jo, S., Kim, T., Iyer, V.G. \& Im, W. CHARMM-GUI: a web-based graphical user interface for CHARMM. J Comput Chem 29, 1859-65 (2008).

85. Wu, E.L. et al. CHARMM-GUI Membrane Builder toward realistic biological membrane simulations. J Comput Chem 35, 1997-2004 (2014).

86. Phillips, J.C. et al. Scalable molecular dynamics with NAMD. J Comput Chem 26, 1781802 (2005).

87. MacKerell, A.D. et al. All-atom empirical potential for molecular modeling and dynamics studies of proteins. J Phys Chem B 102, 3586-616 (1998).

88. Klauda, J.B. et al. Update of the CHARMM all-atom additive force field for lipids: validation on six lipid types. J Phys Chem B 114, 7830-43 (2010).

89. Best, R.B. et al. Optimization of the additive CHARMM all-atom protein force field targeting improved sampling of the backbone phi, psi and side-chain chi(1) and chi(2) dihedral angles. J Chem Theory Comput 8, 3257-3273 (2012).

90. Jorgensen, W.L., Chandrasekhar, J., Madura, J.D., Impey, R.W. \& Klein, M.L. Comparison of simple potential functions for simulating liquid water. J. Chem. Phys. 79, 926-935 (1983).

91. Feller, S.E., Zhang, Y.H., Pastor, R.W. \& Brooks, B.R. Constant pressure molecular dynamics simulations - The Langevin piston method. J. Chem. Phys. 1995, 103, 4613-4621. J. Chem. Phys. 103, 4613-4621 (1995).

92. Darden, T., York, D. \& Pedersen, L. Particle mesh Ewald: An N•log (N) method for Ewald sums in large systems. . J. Chem. Phys. 98, 10089-10092 (1993).

93. Hopkins, C.W., Le Grand, S., Walker, R.C. \& Roitberg, A.E. Long-Time-Step Molecular Dynamics through Hydrogen Mass Repartitioning. J Chem Theory Comput 11, 1864-74 (2015).

94. Tuckerman, M.E., Berne, B.J. \& Martyna, G.J. Reversible multiple time scale molecular dynamics. . J. Chem. Phys. 97, 1990-2001 (1992).

95. Andersen, H.C. Rattle: a "velocity" version of the shake algorithm for molecular dynamics calculations. . J. Comput. Phys. 52, 24-34 (1983). 
96. Ryckaert, J.-P., Ciccotti, G. \& Berendsen, H.J.C. Numerical integration of the cartesian equations of motion of a system with constraints: molecular dynamics of n-alkanes. J. Comput. Phys. 23, 327-341. J Comput Phys 23, 327-341 (1977).

97. Miyamoto, S. \& Kollman, P.A. SETTLE: An analytical version of the SHAKE and RATTLE algorithms for rigid water models. . J. Comput. Chem. 13, 952-962 (1992).

98. Li, Z. et al. Subnanometer structures of HIV-1 envelope trimers on aldrithiol-2-inactivated virus particles. Nat Struct Mol Biol 27, 726-734 (2020).

\section{Acknowledgement}

W.W. acknowledges support from the Institute Universitaire de France (IUF), from the European Union's Horizon 2020 research and innovation programme under grant agreement No. 681137, H2020 EAVI and the platforms of the Grenoble Instruct-ERIC center (IBS and ISBG; UMS 3518 CNRS-CEA-UGA-EMBL) within the Grenoble Partnership for Structural Biology (PSB). Platform access was supported by FRISBI (ANR-10-INBS-05-02) and GRAL, a project of the University Grenoble Alpes graduate school (Ecoles Universitaires de Recherche) CBH-EUR-GS (ANR-17EURE-0003). J.L.N. acknowledges funding from Spanish MINECO (BIO2015-64421-R; MINECO/AEI/FEDER, UE), Spanish MCIU (RTI2018-095624-B-C21; MCIU/AEI/FEDER, UE) and Basque Government (IT1196-19). We thank Miriam Hock and Serafima Guseva for previous contributions to the project, the ESRF-EMBL Joint Structural Biology Group for access and support at the ESRF beam lines, J. Marquez (EMBL) from the HTX crystallization facility, C. Mas and J.B. Reiser for assistance on ISBG platforms.

Author Contributions: W.W. conceived and designed the study. J.L.N. supervised the lipid mixing experiments performed by J.T. A.T. supervised neutralization assays performed by N.F. C.C. and F.D. performed molecular dynamics simulation experiments. D.G. produced proteins, mutants and performed crystallization and pull-down experiments. C.Ca. performed the structural studies and interaction studies. W.W. wrote the manuscript with input from all authors.

Competing interests: The authors declare no competing interests. 
bioRxiv preprint doi: https://doi.org/10.1101/2020.11.12.379396; this version posted November 12,2020 . The copyright holder for this preprint (which was not certified by peer review) is the author/funder, who has granted bioRxiv a license to display the preprint in perpetuity. It is made available under aCC-BY-NC-ND 4.0 International license.

Figures and Tables

Table 1. Pseudovirus neutralization by $2 \mathrm{H} 10,2 \mathrm{H} 10-\mathrm{F}, 2 \mathrm{H} 10-\mathrm{RKRF}$ and bi-2H10. IC50s are indicated in $\mu \mathrm{g} / \mathrm{ml}$.

\begin{tabular}{|l|c|c|c|c|c|c|c|}
\hline & Tier & 2H10 wt & 2H10-F & 2H10-RKRF & Bi-2H10 & 2F5 & VRC01 \\
\hline NL4-3 & 1 & 25.20 & 18.68 & 9.15 & 1.84 & 0.16 & 0.20 \\
\hline MN-3 & 1 & $>50.00$ & 30.38 & 9.36 & 1.39 & 0.03 & 0.06 \\
\hline BaL.26 & 1 & $>50.00$ & 19.38 & 9.63 & 6.05 & 1.21 & 0.13 \\
\hline SF162 & $1 \mathrm{a}$ & $>50.00$ & $>50.00$ & 25.19 & 6.14 & 1.22 & 0.39 \\
\hline SF162P3 & 2 & 22.04 & 13.14 & 6.76 & 1.32 & 1.96 & 0.24 \\
\hline SC422661.8 & 2 & $>50.00$ & $>50.00$ & 27.93 & 3.79 & 1.00 & 0.27 \\
\hline JR-FL & 2 & 44.65 & 16.93 & 6.95 & 1.49 & 0.97 & 0.11 \\
\hline JR-CSF & 2 & $>50.00$ & 21.66 & 10.85 & 2.85 & 1.24 & 0.37 \\
\hline QH0692.42 & 2 & $>50.00$ & $>50.00$ & $>50.00$ & $>50.00$ & 1.20 & 1.21 \\
\hline THRO4156.18 & 2 & $>50.00$ & $>50.00$ & $>50.00$ & $>50.00$ & $>50.00$ & 3.84 \\
\hline
\end{tabular}


A
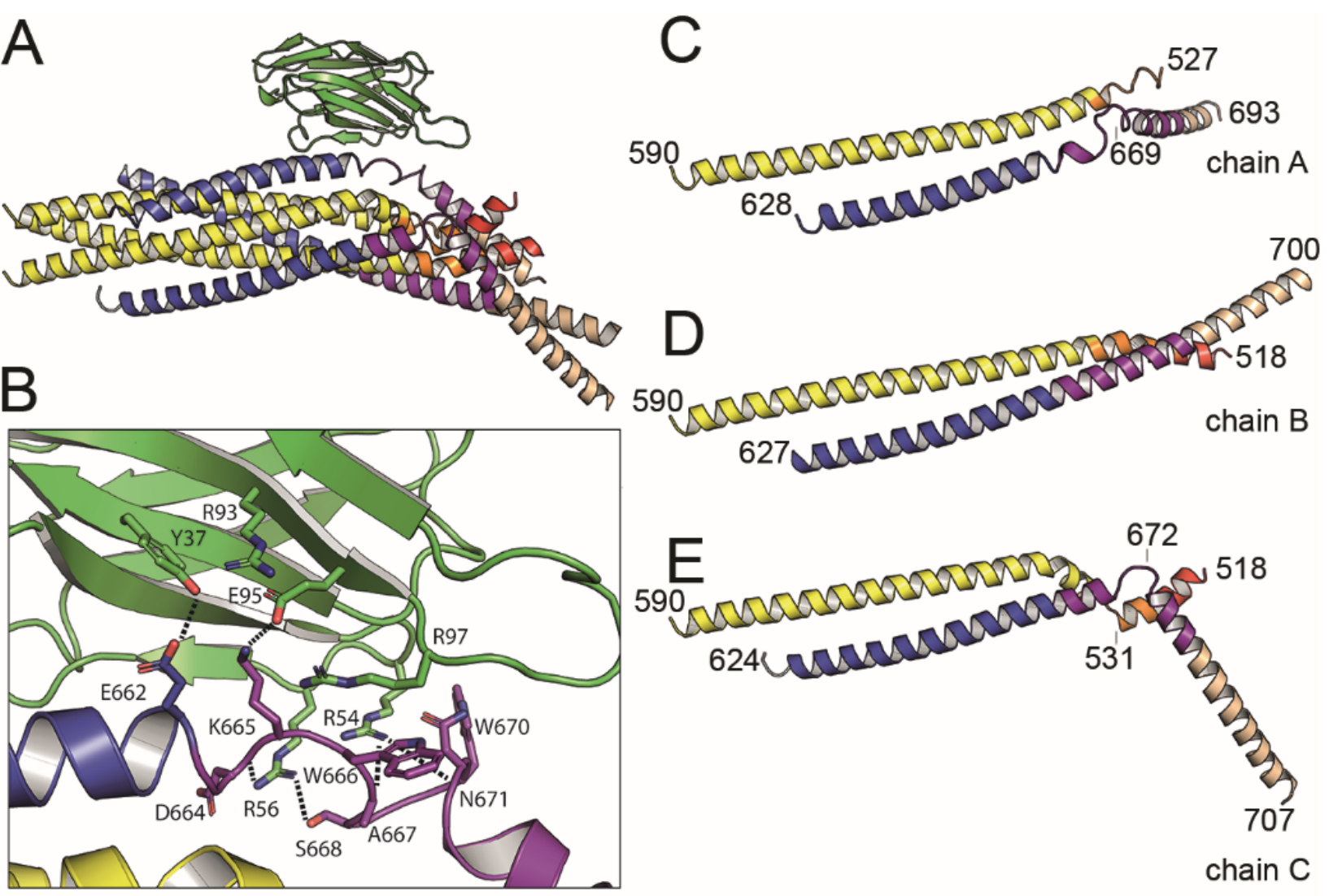

Fig. 1. Crystal structure of gp41FP-TM in complex with $2 \mathrm{H10}$.

A, Ribbon presentation of gp41TM-FP in complex with $2 \mathrm{H} 10$. Color-coding of the different segments is as indicated in the gp41 scheme (Fig. S1A), the 2H10 nanobody is colored in green.

B, Close-up of the interaction of gp 41FP-TM with $2 \mathrm{H} 10$. Residues in close enough contact to make polar interactions are shown as sticks.

C, D, E, Ribbon diagram of the individual protomers named chain A, B and C. Residues within the FPPR and MPER hinge regions are indicated. 
A
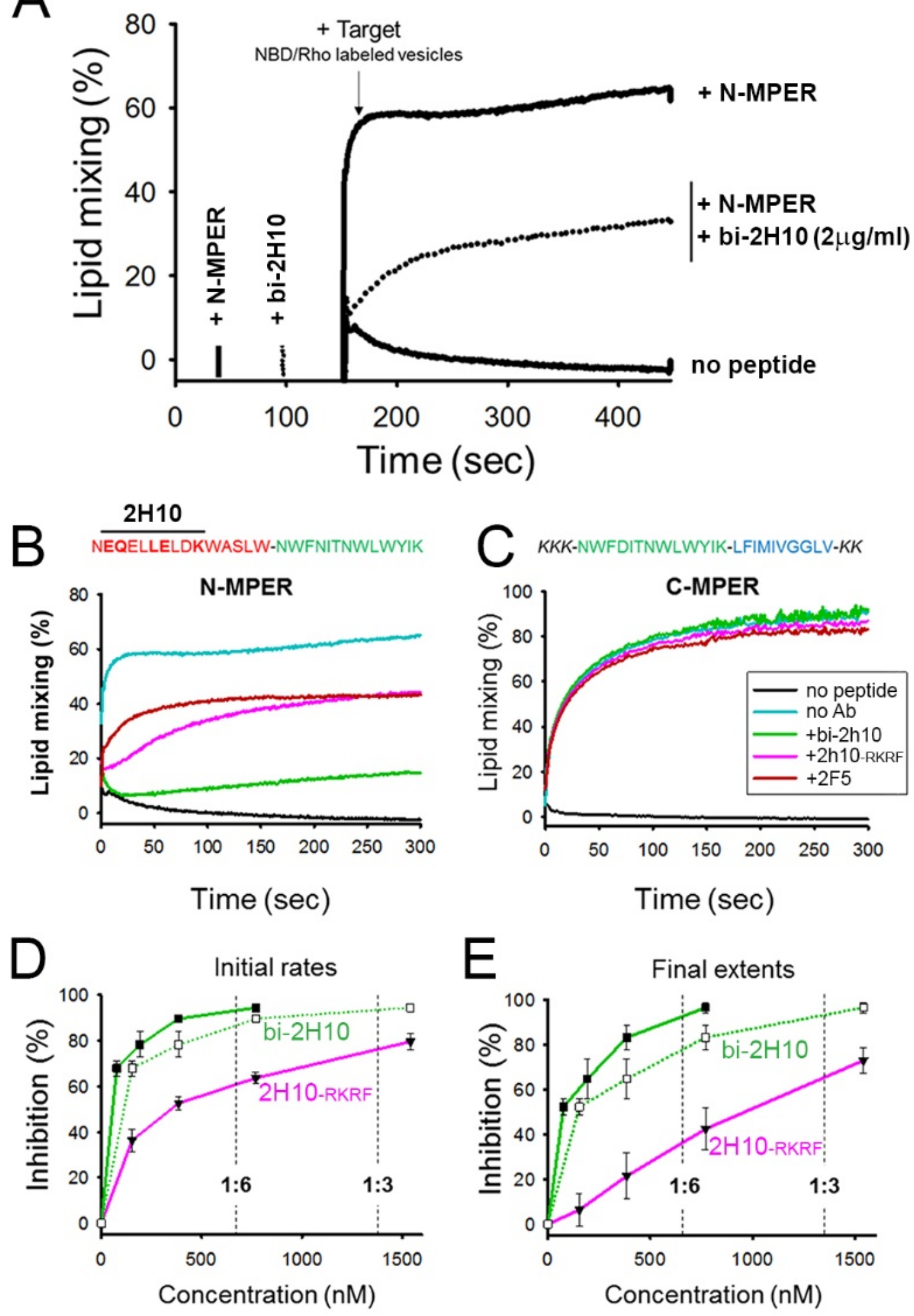

Fig. 2. Vesicle-vesicle fusion inhibition by $2 \mathrm{H10}$, bi-2H10 and 2 F5.

802 (A) Time course of the lipid-mixing assay using fusion-committed vesicles. At time $30 \mathrm{sec}\left({ }^{6}+\mathrm{N}-\right.$ 803 MPER'), peptide (4 $\mu \mathrm{M})$ was added to a stirring solution of unlabeled vesicles (90 $\mu \mathrm{M}$ lipid), and, 
804 after $120 \mathrm{sec}$ (indicated by the arrow), the mixture was supplemented with N-NBD-PE/N-Rh-PE805 labeled vesicles (10 $\mu \mathrm{M}$ lipid). The increase in NBD fluorescence over time follows the dilution of 806 the probes upon mixing of lipids of target and primed vesicles (+N-MPER trace). NBD increase 807 was substantially diminished in samples incubated with bi-2H10 $(2 \mu \mathrm{g} / \mathrm{ml})$ prior to the addition of 808 the target vesicles (+bi-2H10, dotted trace), and totally absent if unlabeled vesicles were devoid of 809 peptide ('no peptide' trace).

810 (B) Left: Kinetic traces of N-MPER-induced lipid-mixing comparing the blocking effects of 2H10811 RKRF, bi-2H10 and Fab 2F5.

812 (C) Absence of effects on lipid-mixing when vesicles were primed for fusion with the C-MPER 813 peptide, devoid of $2 \mathrm{H} 10$ and 2F5 epitope sequences. Antibody concentrations were $20 \mu \mathrm{g} / \mathrm{ml}$ in 814 these assays.

815 (D) Dose-response plots comparing the inhibitory capacities of 2H10-RKRF and bi-2H10 (purple 816 and green traces, respectively). Levels of lipid-mixing 20 or $300 \mathrm{sec}$ after target vesicle injection 817 were measured (initial rates $\mathbf{D}$ and final extents, E) and percentages of inhibition calculated as a 818 function of the $\mathrm{Ab}$ concentration. The dotted line and empty symbols correspond to the effect of bi$8192 \mathrm{H} 10$ when the concentration of the component $2 \mathrm{H} 10$ was plotted. The slashed vertical lines mark 820 the 2 H10-to-peptide ratios of $1: 6$ and 1:3. Plotted values are means \pm SD of three independent 821 experiments. 
A $\quad$ B

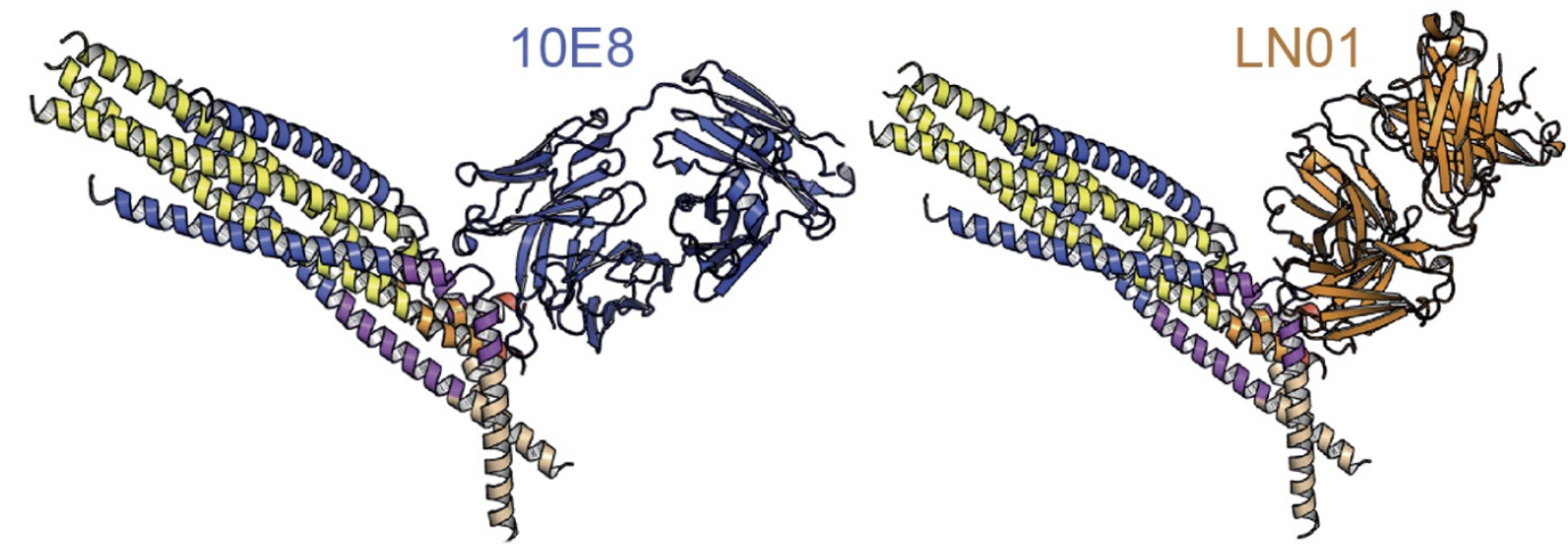

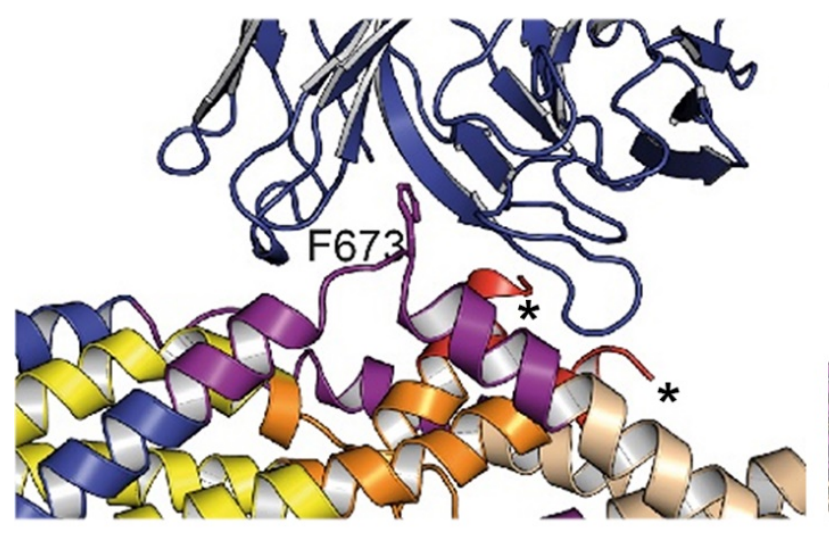

C [gp41 FP-TM] : $0.2-25.6 \mathrm{nM}$

1:1 model, $\mathrm{KD}=0,2+/-0,005 \mathrm{nM}$

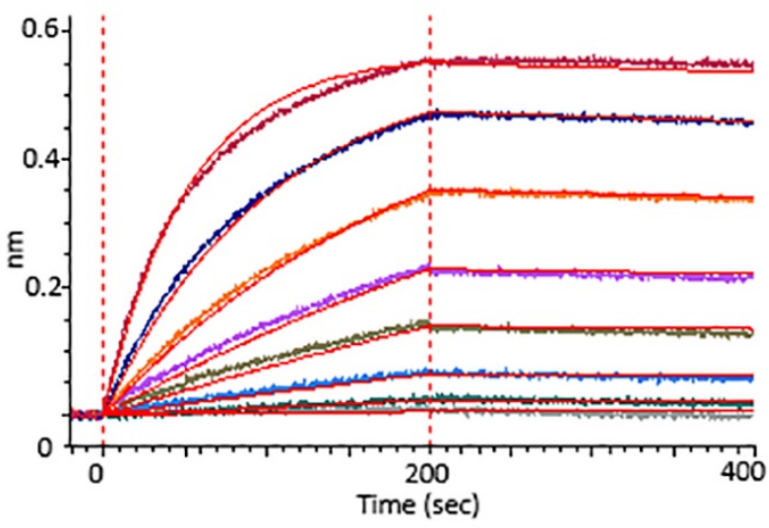

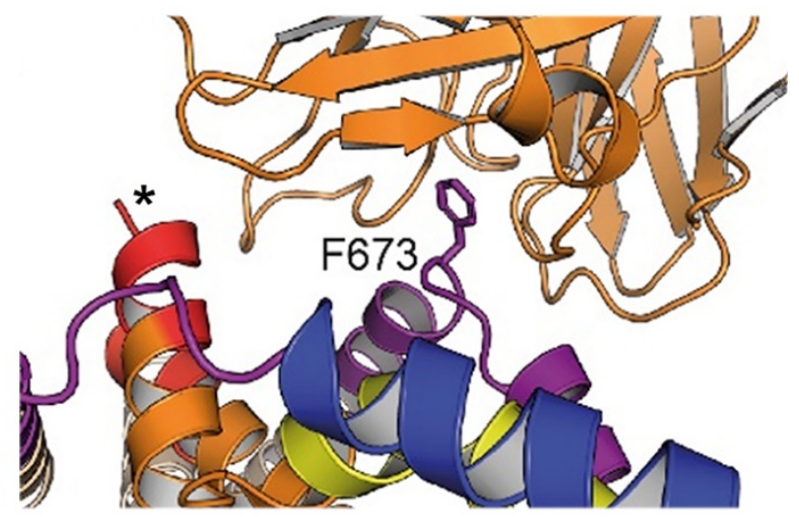

D $\quad$ LN01 IgG

[gp41 FP-TM] : $39-625 \mathrm{nM}$

$\mathrm{KD}=34+/-3,4 \mathrm{nM}$

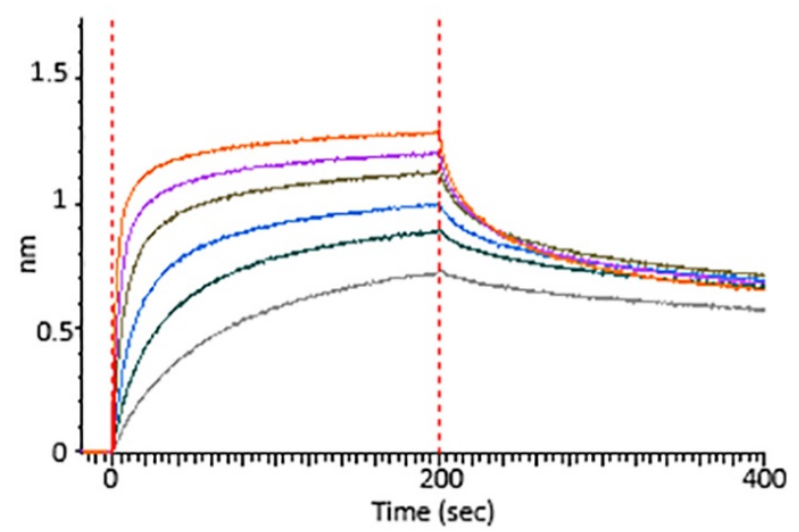

Fig. 3. Gp41FP-TM interaction with bnAbs LN01 and 10E8

A, C $\alpha$ superposition of the MPER peptide structure in complex with LN01 (pdb 6snd) onto chain C-C of gp41FP-TM-2H10 structure. The lower panel shows a close-up of the interaction oriented with respect to gp41 F673. 
$828 \mathrm{~B}, \mathrm{C} \alpha$ superposition of the MPER peptide structure in complex with $10 \mathrm{E} 8$ (pdb 5iq7) onto the 829 corresponding chain C-C of gp41FP-TM. The lower panel shows a close-up of the interaction in 830 the same orientation as in A.

831 C, Bio-layer interferometry (BLI) binding of gp41FP-TM to 10E8 and D, to LN01. 10E8 binding 832 was fit to 1:1 model and for LN01 a steady state model was employed for fitting the data. For 10E8 833 binding, gp41FP-TM was used at concentrations from 0.2 to 25,6 $\mathrm{nM}$ and for LN01 binding 834 gp41FP-TM concentrations ranged from 39 to $625 \mathrm{nM}$. 

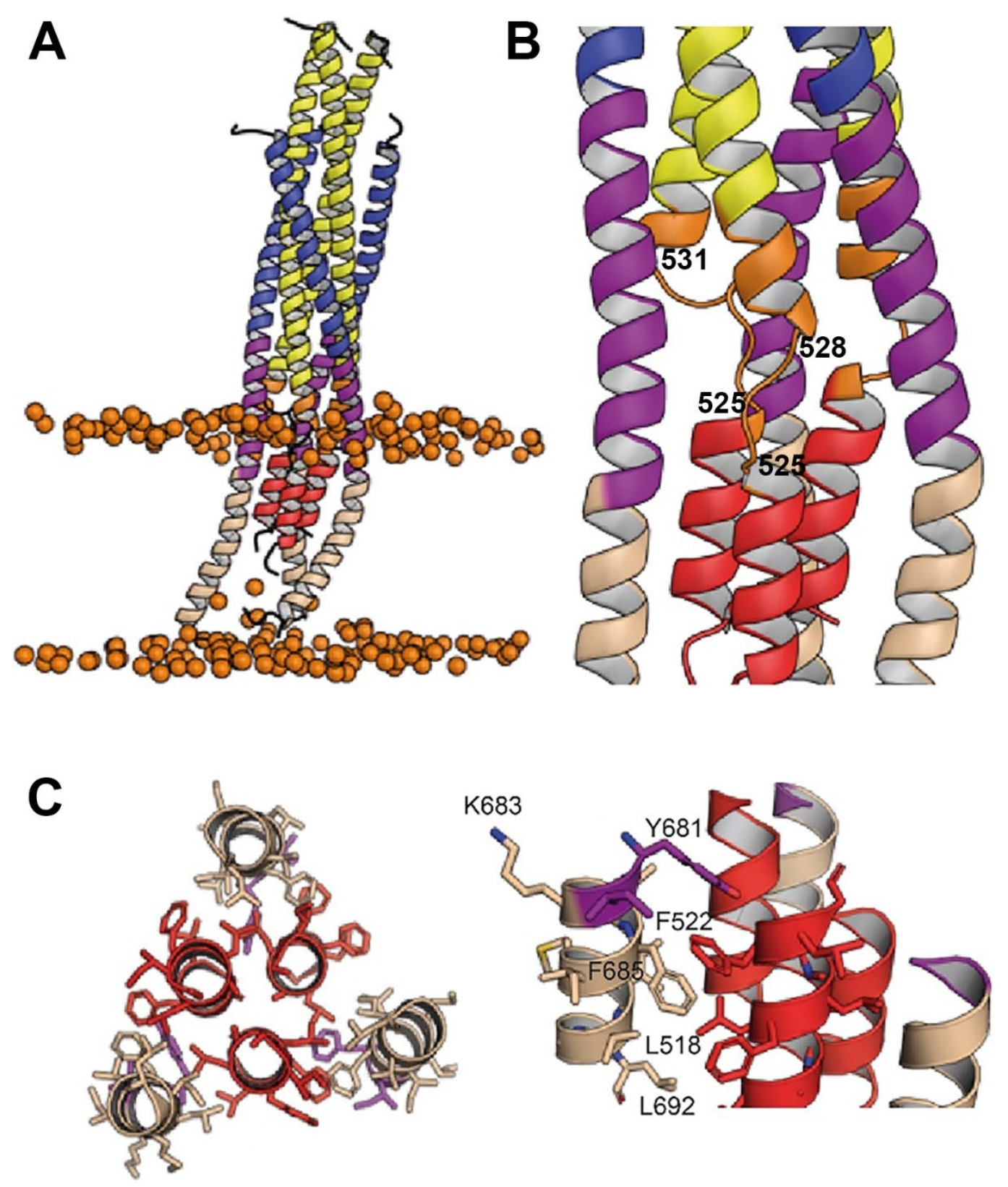

Fig. 4. Interactions within the final post fusion conformation of gp41FP-TM modeled by MD.

A, Model of gp41FP-TM (Fig. S7C) after $1 \mu$ s MD simulation in a bilayer. Phosphate groups of the

840 phospholipids are shown as orange spheres to delineate the membrane boundaries.

B, Close up on the MPER and FPPR flexible regions.

C, Close-up of the interaction of FP (residues 514-524) and TM (residues 681-692) viewed along the three-fold axis from the $\mathrm{N}$-terminus indicating an intricate network of hydrophobic interactions (left panel) and from the side (right panel). Interacting side chains are labeled and shown as sticks. 

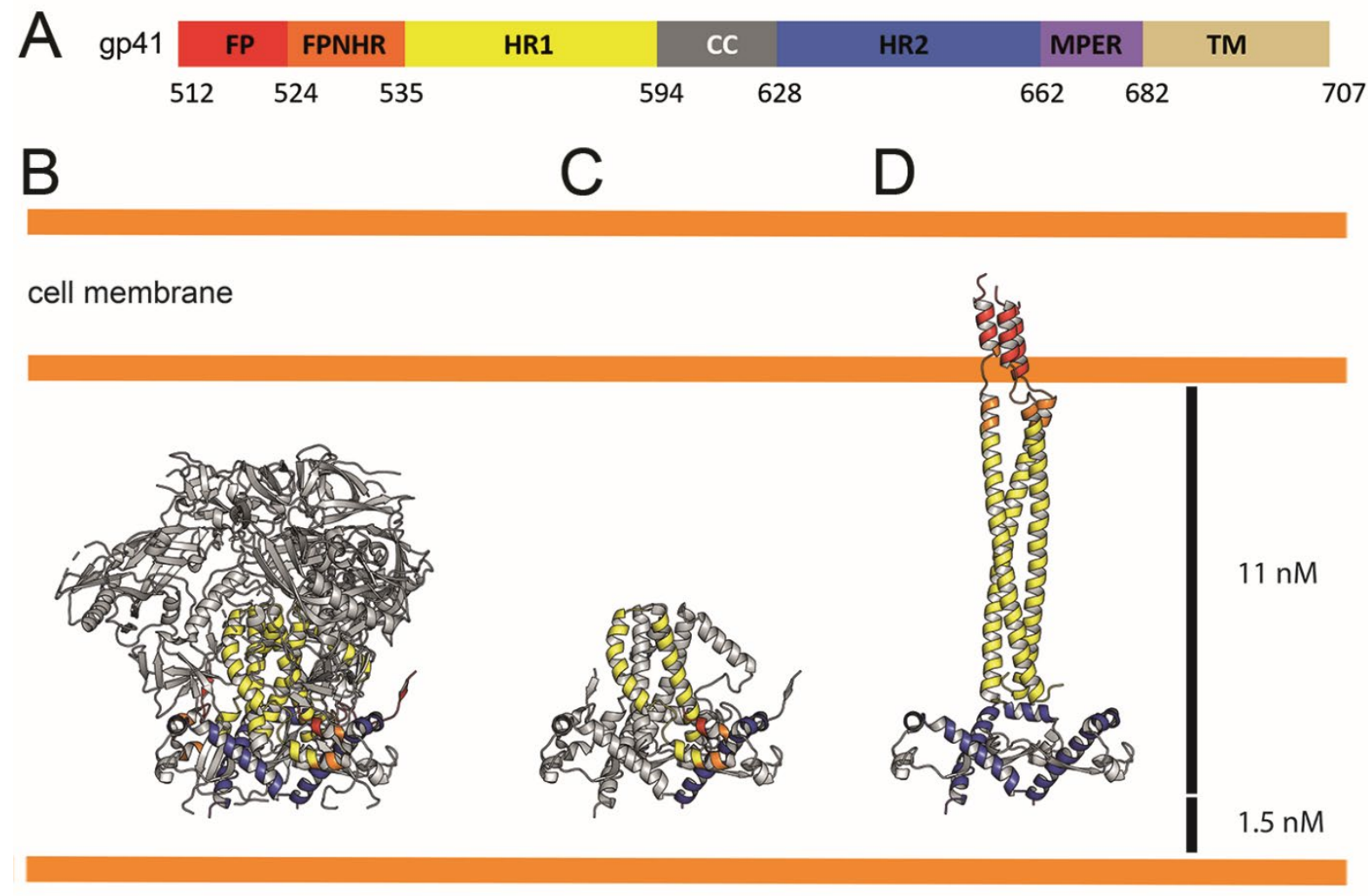

virus membrane

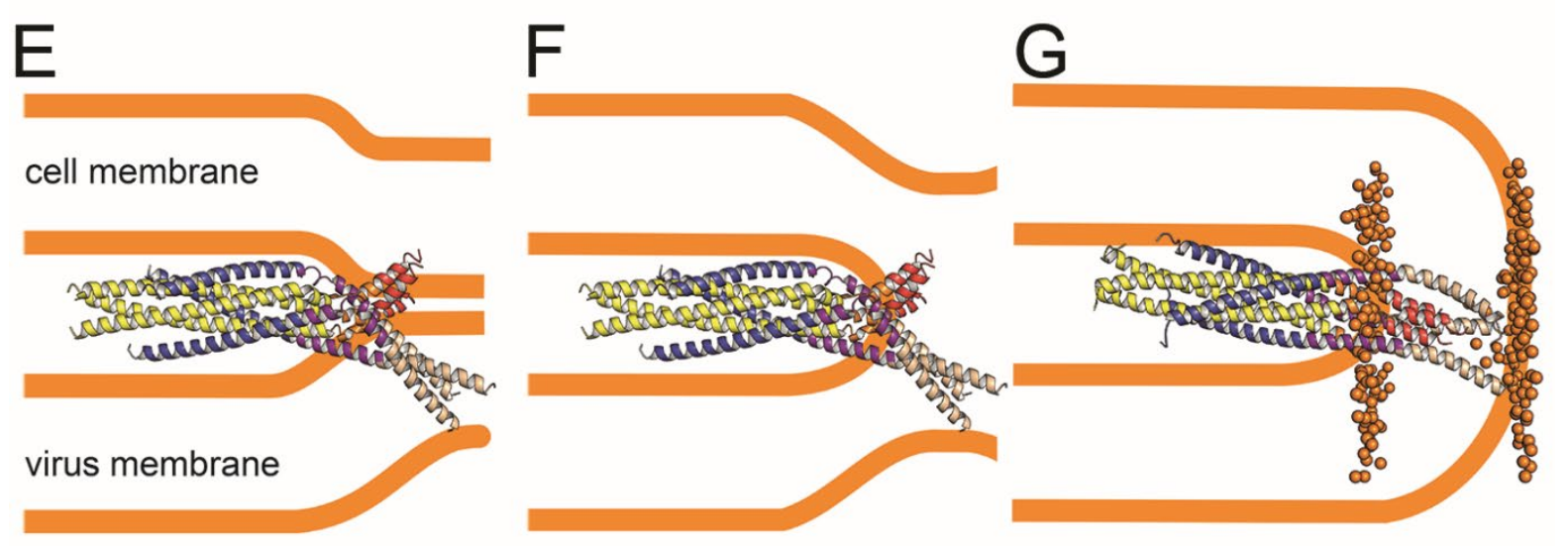

Fig. 5. Conformational transitions of gp41 that lead to membrane apposition and membrane fusion.

849 A, Representation of the different domains of gp41 with the residue numbers delimiting each 850 domain as indicated. The same color code has been used in all the figures.

851 B, Ribbon presentation of the Env prefusion conformation (pdb 5fuu), gp41 is constrained by gp120 852 in its native conformation. The structure of native gp41 lacks the MPER and TM regions. MPER is 853 spanning a distance of $1.5 \mathrm{~nm}^{98}$. 
C, Ribbon of native gp41, one chain is colored according to the sheme in A and the other two chains

855 are shown in grey.

856 D, Binding to cellular receptors CD4 and subsequently to CXCR4/CCR5 induces a series of

857 conformational changes that eventually leads to the dissociation of gp120. During this process,

858 HR1, FPPR and FP will form a long triple stranded coiled coil extending $11 \mathrm{~nm}$ towards the target

859 cell membrane. In a first step HR2 may keep its prefusion conformation in analogy to a similar

860 intermediate, activeted influenza virus HA structure ${ }^{45}$. Alternatively, HR2 may dissociate and form

861 a more extended conformation in agreement with locked gp41 structures bridging viral and cellular

862 membranes that bridge distances of 11 to $15 \mathrm{~nm}^{46}$.

863 E, Bending of HR1 and HR2 will result in the six-helical bundle core structure bringing cellular

864 and viral membranes into close apposition with the 3 FPs anchored in the cellular membarne and

865 the 3 TMs anchored in the viral membrane, the gp41 conformatio represented by the gp41FP-TM

866 structure. This intermedaite gp41 conformation may have brought both membranes into close

867 apposition or may have already induced hemifuison as indicated in $\mathbf{F}$.

868 G, Further reolding of FPPR-FP and MPER-TM results in the final extremely stable post fusion 869 conformation. This thus suggests that rearrangment of the membrane anchors plays crucial roles in 870 lipid mixing, breaking the hemifusion diaphragm to allow fusion pore opening. Boundaries of the 871 lipid layers are shown with orange sphere representing the phosphate atomes of the lipids present 872 in the MD simulation (snapshop taken after $1 \mu$ s MD simuation). 